\title{
Spatial and Temporal Disaggregation of Water Demand and Leakage of the Water Distribution Network in Skiathos, Greece
}

\section{Dimitrios Kofinas ${ }^{1,2}$, Nikolaos Mellios ${ }^{1,2}$ and Chrysi Laspidou ${ }^{1,2, *}$}

1 Information Technologies Institute, CERTH, 6th km Charilaou-Thermi Rd., Thermi 57001, Greece; E-Mails: dimitristheokofinas@iti.gr (D.K.); nikosmellios@iti.gr (N.M.)

2 Civil Engineering Department, University of Thessaly, Pedion Areos, Volos 38334, Greece

* Author to whom correspondence should be addressed; E-Mail: laspidou@iti.gr;

Tel.: +30-24210-74147; Fax: +30-24210-74169.

Published: 6 November 2015

\begin{abstract}
Pressure control management of a water distribution network is considered as an effective approach for the reduction of leakage in the network and for optimized savings in pumping energy. A successful pressure management scheme usually requires single- or multi-feed regulation through Pressure Reduction Valves, the network division in District Metered Areas and dense monitoring of pressure and flow throughout the network. A structural component of this approach would be a hydraulic model which would relate pressure and flow in the network, in accordance to the monitored values. In the ISS-EWATUS project and specifically for the Skiathos, Greece case study, we solve the water distribution network hydraulically using EPANET software, so as to estimate the pressure-map of the island and later link pressure to leakage. The need to zoom-in spatially and temporarily makes the spatial and temporal disaggregation of aggregated water demand, as provided by the water utility, a necessity. We use the daily aggregated water supply time series and consumer quarterly billing data for each water meter, in order to produce approximate, daily water consumption datasets of each household and daily leakage per household. In this article, we present our disaggregation methodology for the production of these data sets.
\end{abstract}

Keywords: water demand modeling; EPANET software; pressure management; water distribution network 


\section{Introduction}

The need to reduce leakages in water distribution networks on one hand and energy consumed for water pumping on the other hand have turned the pressure control management method into the state-of-the-art solution for water companies. The idea is keeping pressure in the network to minimum heights demanded [1]. This results to leakage reduction, since it is directly related to pressure, and to energy consumption reduction, since the higher pressure maintained the more energy is demanded. The demand varies temporarily and spatially depending on the water consumption profiles of the users through day, week, seasons, etc. and the water uses distribution varies throughout the area of interest. The specifications of the case study, in addition to the constraints in terms of budget, lead to a specialized pressure control scheme [2].

The pressure control is implemented with use of Pressure Reduction Valves (PRVs), which adjust their diameters to the water demand anytime. The scheme might be single-feed or multi-feed depending on the deviation of the network in District Metered Areas (DMAs). Large networks with intense spatial variation in latitude and water demand are generally expected to be divided into more DMAs. Each DMA is supposed to retain approximately common characteristics in terms of pressure demand profiles. Single-feed PRV schemes are preferable to more uniform and/or small networks, due to their ease of control and monitoring, even the risk of a failure event is higher to such less flexible schemes [3].

The diameter of the PRV is usually an output of a multi-objective optimization process, which minimizes the leakage and energy consumption variables. The problem could be simplified to a single objective optimization, which minimizes the pressure in the network. Leakage — as well as energy-is expressed as a function of the pressure through variable empirical models [4]. A basic constraint of the optimization process would be the minimum pressure demand of a critical point of the network. This would be the lowest pressure point, either because it is the farthest away from the source, or because it has the highest latitude. It could even be a combination of the two conditions. The critical point, also, might change from time to time, depending on the dynamic consumption profiles variability. Nonetheless, this implies the need to map the pressure demand spatially and temporarily through the network at the highest possible space and time resolution. This can be achieved by mapping the water demand and then using a hydraulic model to calculate the pressure map.

The resulting resolution will determine the effectiveness of the applied network pressure management. Recent established technologies allow network operators to achieve real time management through a Decision Support System (DSS) based on algorithms, containing the leakage model, a forecast model of the consumption, an optimization sub-routine, and the real time monitoring of pressure by sensors installed at the critical point or multiple points [5].

The purpose of this article is to investigate the potential of using daily groundwater pumping data and quarterly consumer billing data in order to produce daily water demand time series for each individual consumer. Additionally, we "assign" a fraction of the network background leakage to each water meter. This way, we perform disaggregation of water demand data sets spatially and temporally in order to heighten the resolution of water consumption and produce a more detailed leakage model for the Skiathos, Greece case study. The final desired spatial resolution would be at the level of the 
node of the hydraulic model, if not the individual consumer, and the desired temporal resolution would be a 6 - to 12 -h window.

\section{Experimental Section}

Skiathos Island, Greece is one of the two case study areas of the EU FP7 funded project ISS-EWATUS. The water distribution network of Skiathos is an aged network with significant leakage, reaching up to $50 \%$ levels of the whole water consumption. The network is currently under reconstruction, works that are quite time-consuming, due to the importance of the maintenance of the traditional street infrastructure, the high touristic activity and other policy related matters. Skiathos has a six-month high touristic activity_-summer water demand peaks up to a point six times multiple the average winter demand [6]. The water uses through the town of Skiathos are almost uniformly distributed and except for households they consist of small hotels and rooms to let, offices, shops and restaurants. The town is hilly and the water distribution system is significantly net-shaped and quite thick in terms of consumers [7].

The small extent of the network and the uniformity of uses allowes for the choice of a single-feed PRV scheme. The whole town comprises approximately 3500 water meters and is considered as a single DMA; pressure control management will be implemented through a single PRV installed downstream the tank that supplies the town with water from a single groundwater drilling. In the future, a booster pump might be added to the pressure control management scheme, cutting off the hilly area of the town into a second DMA, for further localization of the pressure demand constraint.

The available data of water consumption consists of a data set of daily pumped groundwater from a single drilling filling a single tank; this data set is equal to the total daily water consumption of the whole town plus network leakage. Another data set includes quarterly water consumption for each household. The data sets are updated continuously and are currently approximately 5-years long.

For each water meter $k$ and for day $t$ of the trimester tri, the theoretical water demand $d_{k, t, t r i}$ is calculated, which also contains the leakage that theoretically corresponds to the household:

$$
d_{k, t, t r i}=w_{k, t r i} * D_{t, t r i}
$$

where, $D_{t, t r i}$ : The water demand of the whole town for day $d$ of the trimester, or the daily pumped water $w_{k, t r i}$ : The specific weight of each water meter for trimester tri. This weight is calculated as follows:

$$
w_{k, t r i}=\frac{d_{k, t r i}}{\sum_{t} D_{t, t r i}}
$$

$d_{k, t r i}$ : The water demand of water meter $k$ for the whole trimester tri including the leakage that corresponds to that water meter. That is the trimester billing for water meter $k$ with the theoretical leakage percentage added to it.

$$
d_{k, t r i}=d_{k b i l l e d, t r i} *\left(1+a_{t r i}\right)
$$

where, $d_{k b i l l e d, t r i}$ is the trimester tri water meter billing, and

$$
a_{t r i}=\frac{\sum_{t} D_{t, t r i}-\sum_{k} d_{k b i l l e d, t r i}}{\sum_{k} d_{k b i l l e d, t r i}}
$$

Daily water losses are calculated with Equation (5): 
Daily Water Losses $=D_{t, t r i} \frac{\sum_{t} D_{t, t r i}-\sum_{k} d_{k b i l l e d, t r i}}{\sum_{t} D_{t, t r i}}$

The described methodology is also schematically depicted in Figures 1 and 2.

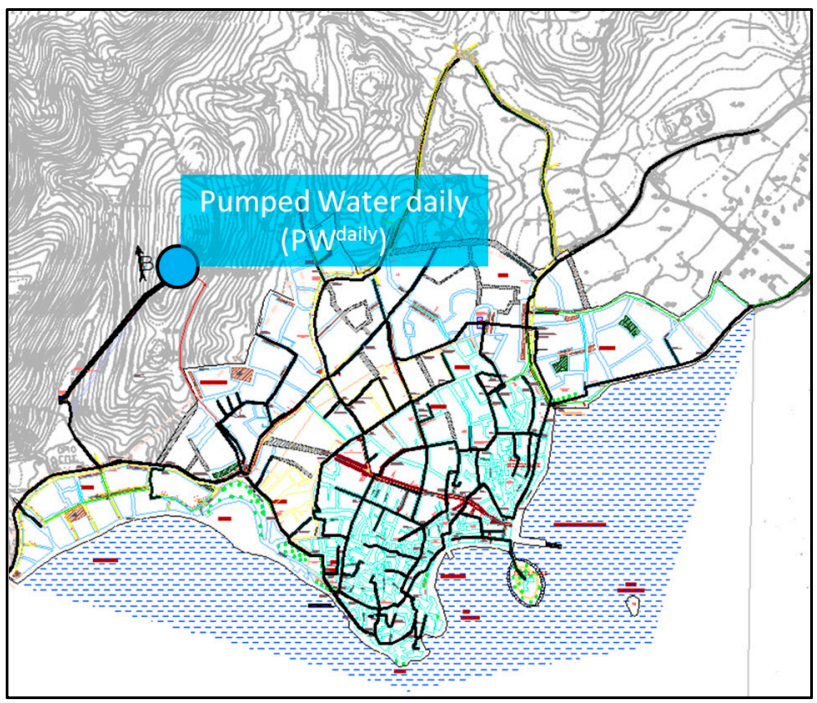

(a)

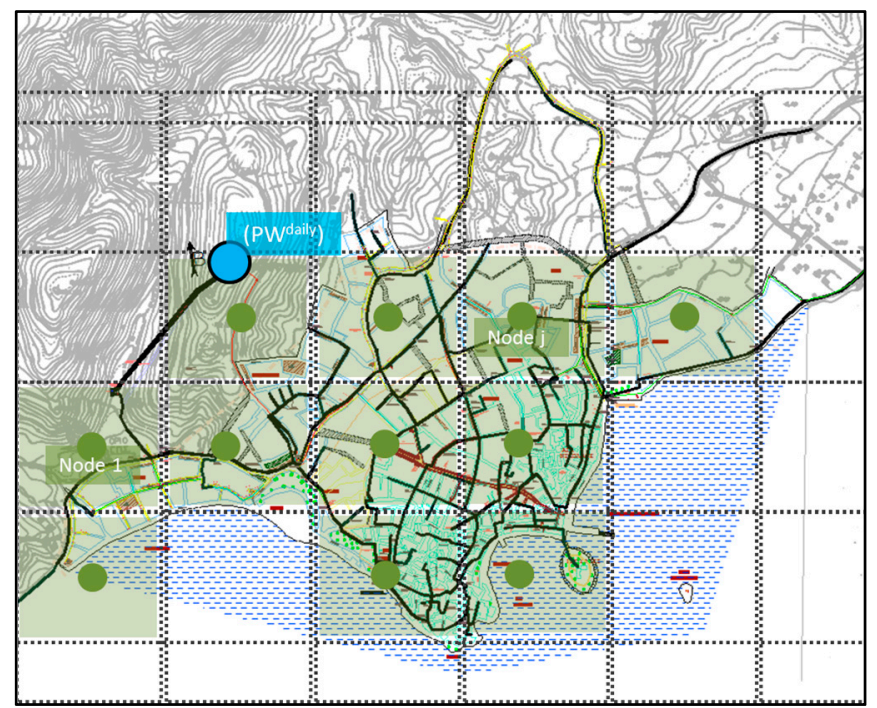

(b)

Figure 1. (a) Water distribution network of Skiathos Island supplied daily by a single drilling; (b) The network is divided into node areas.

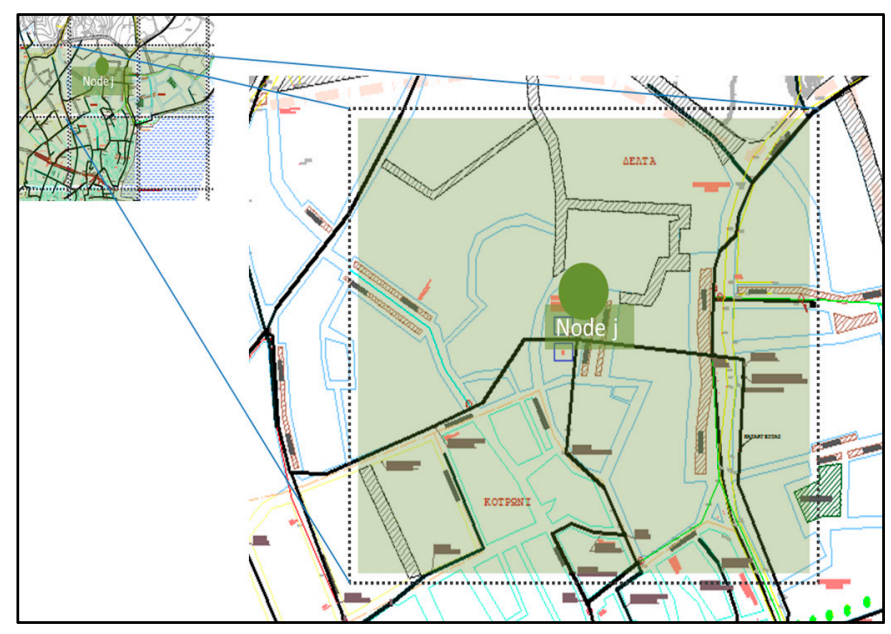

(a)

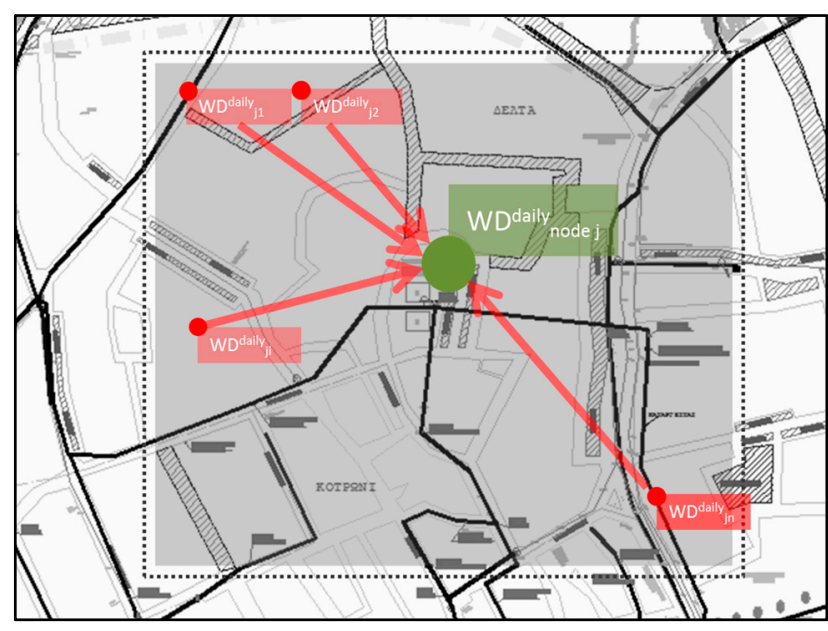

(b)

Figure 2. (a) Each node will be a node of the hydraulic model that will be used to estimate the pressure map out of the water demand map; (b) Each node corresponds to a water demand value that comes out by adding the household water demands that are supplied by the specific node of the network.

\section{Results and Discussion}

Implementing the above-described algorithm, the whole water demand of the town can be distributed to theoretical household demands including (or not) the leakages that "correspond" to the specific households with use of trimester household consumption weights. Of course, we do not expect 
this estimation to be accurate, since throughout a trimester the consumption profile of a household might change dramatically from one day to the next. However, once the individual consumer daily demands are added at a node level (the required granularity for the creation of time series to be input in EPANET software for the hydraulic solution of the network), the error becomes less significant, since a node will include multiple consumers, even as many as a hundred. The expected divergences of the estimated and the actual node water demands will be revealed by comparing estimated data to monitoring data obtained by sensors; these are located, at a minimum, at critical points in the network, or at several points throughout the network. These divergences will be eliminated through a successful calibration and through an optimization process that involves multiple runs until convergence is reached.

Using the methodology described above the temporal resolution of the household water demand has zoomed in from a trimester level into a daily level. Once these demands are added at a node level, the water demands needed as inputs to the hydraulic model will be available allowing for the pressure mapping of the network. Moreover the daily water demand of the whole town is spatially disaggregated into node level. Respectfully, the leakage is also disaggregated the same way. An interesting side outcome of the process is the spatial distribution of the leakage. This gives the potential to investigate the construction of a leakage model further than the literature suggested empirical leakage models.

\section{Conclusions/Outlook}

Limited data availability is a major obstacle in the implementation of any pressure management scheme in water distribution networks, since it usually involves water demand forecasting at a level of relatively fine granularity. In "low-tech" situations where smart meters are not available at the consumer level and customer billing is done manually with bills being issued once every three months or longer, data scarcity becomes a serious limitation. In this article, we presented a methodology that can be useful in disaggregating bulk town water supply data to the level of individual consumer. The methodology, although not accurate, it provides a way to deal with the lack of data and produces time series that can be refined later, as sensors and smart meters becomes more widely available.

\section{Acknowledgments}

This work was supported by the project ISS EWATUS-Integrated Support System for Efficient Water Usage and Resources Management - which is implemented in the framework of the EU $7^{\text {th }}$ Framework Programme, Specific programme Cooperation Information and Communication Technologies; Grant Agreement Number 619228.

\section{Author Contributions}

C.L. conceived the disaggregation algorithm idea, while D.K. and N.M. developed it. D.K. and N.M. designed and implemented the algorithm with data from the Skiathos Water Company. C.L. is the Work Package leader in the ISS-EWATUS project. 


\section{Conflicts of Interest}

The authors declare no conflict of interest.

\section{References}

1. Ulanicki, B.; Bounds, P.; Rance, J.; Reynolds, L. Open and closed loop pressure control for leakage reduction. Urban Water 2000, 2, 105-114.

2. Jowitt, P.W.; Xu, C. Optimal valve control in water-distribution networks. Journal of Water Resources Planning and Management 1990, 116, 455-472.

3. AbdelMeguid, H.; Ulanicki, B. Pressure and Leakage Management in Water Distribution Systems via Flow Modulation PRVs. Water Distribution Systems Analysis 2010, 1124-1139.

4. Giustolisi, O.; Savic, D.; Kapelan, Z. Pressure-driven demand and leakage simulation for water distribution networks. Journal of Hydraulic Engineering 2008, 134(5), 626-635.

5. Laspidou, C. ICT and stakeholder participation for improved urban water management in the cities of the future. Water Utility Journal 2014, 8, 79-85.

6. Kofinas, D.; Mellios, N.; Papageorgiou, E.; Laspidou, C. Urban Water Demand Forecasting for the Island of Skiathos. Procedia Engineering 2014, 89, 1023-1030.

7. Laspidou, C.; Papageorgiou, E.; Kokkinos, K.; Sahu, S.; Gupta, A.; Tassiulas, L. Exploring Patterns in Water Consumption by Clustering. Procedia Engineering 2015, 119, 1439-1446.

(C) 2014 by the authors; licensee MDPI, Basel, Switzerland. This article is an open access article distributed under the terms and conditions of the Creative Commons Attribution license (http://creativecommons.org/licenses/by/3.0/). 